\title{
O PROGRAMA DA SAÚDE DA FAMÍLIA COMO ESTRATÉGIA DE ATENÇÃO BÁSICA PRIMARIA PARA O SISTEMA ÚNICO DE SAÚDE
}

\author{
LUANA VEDANA ${ }^{1}$
}

\section{Resumo}

Este artigo tem por objetivo tratar sobre importância do Programa da Saúde da Família, hoje conhecido como Estratégia da Saúde da Família. Sobre a importância da ESF para a reorganização do Sistema Único de Saúde. Procurando compreender melhor como a ESF pode auxiliar a Atenção Básica Primaria, sendo um modelo de assistencialismo, mais próximo das famílias. Foi realizada uma pesquisa bibliográfica acerca do tema "O Programa da Saúde da Família Como Estratégia de Atenção Básica Primaria para o Sistema Único De Saúde”. Como o auxilio de todos os integrantes da equipe, especialmente dos Agentes Comunitários de Saúde, podem colaborar para que haja um melhor atendimento das famílias, percebendo seus problemas e possíveis doenças, promovendo promoções de prevenção e encaminhando para seus devidos tratamentos.

Palavras-chave: Estratégia da Saúde da Família; Sistema Único de Saúde; Atenção Básica.

\section{INTRODUÇÃO}

O presente estudo faz uma contextualização sobre o modelo assistencial de saúde no Brasil, desde a implantação do modelo da estratégia saúde da Família (ESF), que viabilizou os conceitos de atenção primária que surgiram como estratégia política. Dessa forma podese perceber a procura por novos modelos assistencionais, vindos de um momento históricosocial, onde o movimento da reforma do setor saúde, buscou uma assistencia que se adequa-se as necessidades de saúde da população.

Assim o setor da saúde, precisou se organizar para ser mais igualitario e humano, considerando que a saúde poderia elevar-se em qualidade, se as condições de vida dos usuarios fossem melhoradas.

Uma alternativa significativa seria a Estratégia de Saúde da Família (ESF), com

\footnotetext{
${ }^{11}$ Graduação em Enfermagem, especialização em Saúde da Família , Instituto Cultus do Grupo Faveni. vedana.luana@hotmail.com
} 
vistas para atender o que foi disposto na Cosntituição Brasilieira sobre a saúde, e aos principior do Sistema Único de Saúde (SUS).

A Estratégia de Saúde da Família é apresentada como uma maneira de reestruturar a atenção primária partindo de um conjunto de ações combinadas com os princípios do Sistema Único de Saúde (SUS). O principal objetivo da ESF é levar a saúde para mais proximo da família, e dessa forma melhorar a qualidade de vida da população brasileira. Além de priorizar as ações do cidadão e da família de maneira integral e contínua.

\section{DESENVOLVIMENTO}

\subsection{O Sistema Único de Saúde na política de saúde brasileira}

Na década de 90 a necessidade da Estratégia de Saúde da Família (ESF) juntamente com o desenvolvem-no do Sistema Único de Saúde o SUS, causou uma busca pela construção de algum modelo de assistencia a saúde que buscasse ampliar o conceito de saúde e da sua integralização. Procurando criar uma rede de atenção à saúde, onde fosse possível integrar as diversas instituições de saúde, e dar continuidade a atenção básica já oferecida ao indivíduo, facilitando o fluxo de pacientes pelo sistema. Desta maneira, percebeu-se que poderia ser criado um modelo de atenção diretamente ligado aos indivíduos e organizado de forma a prestar um serviço de qualidade e resolutiva.

Estes modelos referem-se a forma que estão dispostos em determinado local e tempo, seriam estas as ações de atenção a saúde. Eles ainda evidenciam as formas de organização e articulação dos métodos de trabalho e dos diversos recursos existentes, suprindo as necessidades em saúde de um determinado grupo. Paim 2001; Silva Junior e Alves, 2007, afirmam que as expressões logicas que guiam as ações possuem racionalidades. Os moldes são relacionados com o contexto socioeconômico, com as dimensões politicas e éticas, e evidenciam os métodos de concepção a saúde.

Ainda de acordo com Paim, 2001, os modelos que buscam a integralidade e pela resolução da assistência precisam analisar e identificar os problemas de saúde da população, focando no usuário e superando as perspectivas racionais. A necessidade da ESF e do SUS evidenciam a procura por este novo modelo centrado na integralização da atenção, mesmo que a estratégia seja problematizada como um programa com poucos custos e focada nas perspectivas, segundo Franco e Merhy (2003) e também Soares (2005). 
Anterior ao SUS existia o modelo chamado "medico assistencial privatista" tinha por característica a prestação de uma assistência curativa, especializada e individual, prestada pelo médico no ambiente hospitalar e ambulatorial, com o uso de equipamentos e procedimentos com altos valores. Por ter um valor alto não garantia acesso a toda população.

O alto custo e a pouca eficiência deste modelo, fez com que o Brasil na década de 70, e também com influencia vinda de âmbitos internacionais, fizesse uma reflexão sobre a sua organização dos serviços de saúde, e captando suas principais dificuldades e sua necessidade de reformulação do sistema (SILVA JUNIOR e ALVES, 2007).

O Movimento da Reforma Sanitária evidenciou os problemas do modelo de saúde e lançou um novo formato para a politica de saúde. O SUS, depois de tratado na Constituição Federal de 1988, passou a ser um direito de todos e como dever do Estado, dessa forma ampliou o próprio entendimento do processo saúde-doença. Dessa forma a saúde passou a ser relacionada com a qualidade de vida, considerando os diversos fatores sociais, econômicos e culturais que afetam no adoecimento do ser e no coletivo (BRASIL,1988).

Na década de 90 com houve a impletanção das Leis Orgânicas da Saude (LOS) 8.080 e 8.142, as quais regulamentaram importantes instrumentos legais do SUS. Estabelecendo os valores e o funcionamento do SUS, destacando a universalidade, que afirma que todo o individuo brasileiro tem direito de acesso aos serviços públicos de saúde. A equidade afirma que as diferenças econômicas e sociais não põem ser empecilhos para o uso dos serviços de saúde. A integralidade afirma que o individuo dever ser visto de forma integral, onde a assistencia a saúde dever entendida como um conjunto tanto para meios individuais como coletivos. A descentralização é a divisão das responsabilidades entre os governos. A Hierarquização e regionalização, diz que as unidades precisam ser organizadas em níveis (SISTEMA ÚNICO DE SAÚDE, 2008).

De acordo com Faria et al, (2009) a organização e a gestão dos processos de trabalho nos serviços de saúde, especialmente, no trabalho das equipes de saúde da família, formando em um dos eixos centrais na reorganização da atenção à saúde no SUS.

\subsection{O Programa de Saúde da Família}

Com este novo modelo de atenção à saúde, por meio da descentralização, a construção de uma rede de serviços, onde os pontos devem ter como foco a Atenção Primaria à saúde 
(APS), onde ela serve como uma porta de entrada do sistema de saúde, tornando-se o primeiro nível de contado da população com as ações e serviços de saúde. De acordo com Corbo et al (2007) esta foi uma estratégia necessária para a melhora das condições de saúde para a população. Partindo destas considerações e procurando consolidar o modelo de atenção a saúde, em 1994 o Ministério Publico lançou o Programa Saúde da Família (PSF), hoje denominado ESF, onde veio para implantar convênios com o Ministério Publico, os estados e os municípios, reorganizando o SUS.

Conforme afirmam Fernandes e Seclen (2004), a Estratégia de Saúde da Família desponta como inovadora e reestruturadora dos serviços e ações de saúde, ao ultrapassar uma visão fragmentada dos indivíduos para que ocorra um entendimento completo nas dimensões individuais e coletivas, ou seja, o resgate das medidas generalista, onde o entendimento do processo saúde-doença a que encontram-se expostos os indivíduos, precisar ser repensada de maneira mais ampla, de acordo com a realidade da comunidade.

A implantação do modelo de estratégia saúde da família para organizar o modelo assistencial, tem por objetivo: oferecer assistencia integral, continua, de resultado, e de boa qualidade, para as necessidades da população. Intervir sobre os fatores de risco que a população se encontra; humanizando as práticas de saúde por meio de vínculos entre os profissionais de saúde e a população; organização dos serviços e da produção social da saúde (COSTA e CARBONE, 2004).

Segundo Monnerat (2007) a proposta do Programa também era incorporar os princípios básicos do sistema. Desenvolvendo ações de saúde partindo de uma equipe de saúde, que trabalhasse com o cadastramento e acompanhamento da população em determinada área.

A equipe de saúde da família deve ser formada por um médico de família, um enfermeiro, uma auxiliar de enfermagem, além de quatro a seis agentes comunitários de saúde. Cada equipe pode contar com mais algum profissional de saúde de acordo com o que o município achar necessário. De acordo com Costa e Carbone (2004), cada equipe é responsável por uma determinada área, atendendo as famílias ali existentes. Onde cada equipe ira realizar o cadastramento, por meio das visitas domiciliares. A hierarquização e a integralidade em relação a unidade de saúde da família esta inclusa no primeiro nível de ações e serviços de saúde, a qual esta inserida na atenção primaria.

O papel apresentado pelos Agentes Comunitários de Saúde é muito importante para alcançar os objetivos da ESF, pois além de profissional da saúde ele é morador da comunidade atendida. Representando um elo entre a equipe e a população daquele território, 
combinando o saber cientifico com o saber popular. Além de desenvolver um trabalho importante de educação em saúde desenvolvendo de maneira externa a unidade, nas famílias sob sua responsabilidade. Facilitando o acesso as divulgações das ações e serviços da unidade de saúde, estreitando os vínculos da população com a equipe, desenvolvendo um trabalho de prevenção da promoção da saúde (PNAB, 2011).

De acordo com Silva et al, (2005) afirma que o fato desse profissional morar na mesma comunidade em que trabalha possibilita o desenvolvimento de uma integração muito boa entre a comunidade e a equipe de saúde. Ao vivenciar a realidade da população, ele consegue fazer uma ampla leitura e pode levar estas questões para a equipe. Essa interdisciplinar é essencial no trabalho da ESF.

Dessa forma, a formação e a educação permanente, precisam fazer parte da ESF assim ela poderá alcançar os objetivos que o Ministério da Saúde lhe propôs, incluindo desde os Agentes Comunitários como os demais membros da equipe. Teixeira e Nunes (2007) afirmam que a composição da equipe precisa ser repensada e agregar novos profissionais. Para não correr o risco de não conseguir atender da forma correta todas as demandas.

\subsection{A Atenção Primária à Saúde e os usuários na ESF}

A definição da atenção primária é o primeiro contato ou a entrada no sistema de saúde, e ao mesmo tempo em que constitui um nível próprio de atendimento. Com a função de garantir atenção as maiores necessidades de saúde e direcionar aos outros níveis de atendimento. Para resolver as necessidades básicas de saúde, no caso das demandas sanitárias, as relacionadas a algumas ações clinicas e as demandas tipicamente clinicas de prevenção e recuperação (SCHRAIBER, 1998). É necessário que a unidade de saúde da família tenha fácil acesso, sem barreiras financeiras, geográficas e culturais, aspectos estes encontrados no PSF.

Para ilustrar tal importância ao SUS, o Programa Saúde da Família (2008), descreve o programa como: (...) estratégia prioritária para a reorganização da Atenção Básica no Brasil, reafirmando os princípios básicos do SUS: universalização, equidade, descentralização, integralidade e participação da comunidade, mediante o cadastramento e a vinculação dos usuários.

Desta forma, o programa contribui com o processo de desospitalização e humanização do SUS, visando à valorização dos aspectos que influenciam a saúde das pessoas fora do ambiente hospitalar. 
A ESF na visão de Viana e Dal Poz (1998) representa a primeira tentativa de reformulação, do modelo de APS. Ela seria um alargamento da APS, incorporando as práticas preventivas, curativas e educativas mais próximas do dia-a-dia da população e dos grupos mais vulneráveis.

O modelo de APS na ESF, constituem quatro embasamentos fundamentai: a globalidade, a continuidade, a longitudinalidade e o vínculo. Os quais devem estar focados na comunidade e na busca de soluções para seus problemas (BRASIL, 1997).

O Ministério da Saúde especifica que para a ESF, ser realmente uma estratégia familiar, ela dever ser responsável por: ter conhecimento da realidade das famílias que é responsável, por meio do cadastramento de cada uma delas e do diagnostico de suas características sociais, epidemiológicas e demográficas; identificar os problemas de saúde e as possíveis situações de risco; elaborar, juntamente com a comunidade, um plano local para o enfrentamento das causas dos determinantes de processos de saúde e doença; proporcionar e prestar assistência de maneira integral, respondendo de maneira contínua e racional a demanda organizada e espontânea na Unidade de Saúde da Família, na comunidade, nas casas e no acompanhamento dos atendimentos ambulatoriais e hospitalares, apresentar ações educativas e sensitivas para o enfrentamento dos problemas de saúde, ações estas que as equipes devem ser preparadas (BRASIL, 2000).

Para Starfield (2002), conduzir os serviços de saúde no contexto familiar, possibilita um maior conhecimento do grupo de pessoas; dipor de um espaço para a avaliação dos problemas dos usuários; é necessario para a tomada de decisão para que ocorra uma intervenção apropriada, porque as familias podem dicordar da sua capacidade de executar outros tratamentos e manejo das estratégias.

Ao tratar da demanda espontânea referem-se a um determinado tipo de atenção, a qual deve ser aplicada as condições e aos eventos, inesperados, solicitada pelo individuo em que sentir algum sintoma ou sinal inesperado. O serviço de saúde precisa planejar-se para atender esse tipo de situação. Para minimizar essa demanda é preciso implantar ações de melhoria da atenção programada as condições crônicas, ou seja, ofertar mais atenção programada aos portadores dessas enfermidades, principalmente nas ESFs (MENDES, 2012).

Ao nível de atenção preventiva, a Estratégia de Saúde da Família propôs o desenvolvimento de práticas de educação em saúde direcionadas para melhorar o autocuidado dos indivíduos. Onde todos os profissionais em seus atendimentos a pacientes doentes e não 
doentes devem desenvolver. Dessa forma, se percebe que as práticas educativas da ESF, não precisam ser desenvolvidas em espaços restritos, pode ser desenvolvida nas famílias e assim aumentando as chances de promoção da saúde (BRASIL, 1997).

De acordo com Ozório (2000) nas ESFs, as atividades educativas são realizadas de maneira de grupos de operativos. Trabalhando de forma coletiva, enraizando o conhecimento. Esses grupos operativos propõem reuniões de indivíduos com as mesmas dificuldades, para que juntos troquem experiências e com estas informações repassadas para os coordenadores do grupo, consigam transformar essas informações em conhecimentos reais.

Viana e Dal Poz (1998) afirmam que o papel da ESF é muito importante na reestruturação do SUS, pois evidencia as fragilidades e as limitações do modelo tradicional. Afirma que o programa foi uma importante ferramenta na organização dos sistemas locais de saúde, a medida acabou impondo alguns requisitos para as assinaturas de convênios; também auxiliou na pressão ao processo de descentralização, por meio da exigência no enquadramento na Norma Operativa Básica (NOB - 93), com recebimentos de recursos; atuou sobre as mudanças nos critérios de pagamento do sistema, chegando à institucionalização, por meio da NOB -96 .

Afirmam ainda que é evidente o papel do programa no avanço das maneiras organizativas da população, além de incentivar mais a organização e a institucionalidade dos meios de representação da comunidade, ou seja, possibilitando uma maior participação e controle destas ações públicas.

Rosa e Labate (2005) afirmam que:

Seja como for, acredita-se que a ESF tem potencialidades como estratégia para mudança do modelo assistencial, mas verifica-se a necessidade de flexibilizar as tarefas estabelecidas para o seu funcionamento, assim como conscientizar não somente os profissionais de saúde e universidades para o aprimoramento de conhecimentos no que se refere a uma abordagem às famílias, trabalho em equipe, assistência humanizada, mas, também, conscientizar os gestores e a população da importância da sua participação no planejamento das ações uma vez que é um trabalho de parceria ESF/Família/Comunidade (Rosa e Labate, 2005, p. 1033).

A ESF quando bem planejada e com a equipe preparada auxilia nas necessidades do sistema de saúde brasileiro, além de minimizar os problemas relacionados ela traz vários benefícios. 


\section{CONCLUSÃO}

Contudo, as Estratégias de Saúde da Família em seu trabalho tem como uma de suas prioridades operacionais a lógica da vigilância em saúde que seria a compreensão do processo saúde doença em uma determinada localidade sócio-político-geográfica. Esse planejamento possibilita a compreensão das demandas ditas ao território, permitindo um planejamento adequado das intervenções que se voltam tanto para os indivíduos, quanto para as famílias e a comunidade.

Percebe-se que ocorre uma transformação no modelo assistencial, assim torna-se essencial que haja um trabalho em equipe interdisciplinar e a inclusão familiar seja um objetivo da atenção básica indo além do cuidado individualizado, focado na doença.

A ESF surgiu como uma proposta para melhorar o modelo de assistência e também viabilizar e reorganizar a atenção primaria à saúde, introduzindo o conceito de interdisciplinaridade para sistematizar suas praticas, através de diferentes categorias do campo de saúde, proporcionando maior eficiência nas prevenções e promoções de saúde na comunidade.

\section{REFERÊNCIAS}

BRASIL. Constituição de 1988. Constituição da República Federativa do Brasil. Brasília Senado, 1988.

BRASIL, M.S. Saúde da família: uma estratégia para reorientação do modelo assistencial, Brasília, 1997.

BRASIL. Ministério da Saúde. Programa Saúde da Família. 2000.

CORBO, Anamaria D’Andrea; et al. Saúde da Família: construção de uma estratégia de atenção à saúde. In: MOROSINI, Marcia Valeria; CORBO, Anamaria D’Andrea. Modelos de atenção e a saúde da família. Rio de Janeiro: EPSJV/Fiocruz, 2007.p. 69-106.

COSTA, E. M. A.; CARBONE, M. H. Saúde da Família: uma abordagem interdisciplinar. Rio de Janeiro: Rubio, 2004.

FARIA, H. P.; WERNECK, M. A. F.; TEIXEIRA, P. F. S.; SANTOS, M. A. Processo de trabalho em saúde. 2 ed. Belo Horizonte: Nescon/UFMG, 2009. 68p. 
FERTONANI, H. P. O desafio de construir um novo modelo assistencial em saúde: reflexões de trabalhadores do PSF de uma unidade de saúde de Maringá PR. 2003. Dissertação (Mestrado em Enfermagem) - Universidade Federal de Santa Catarina, Florianópolis, 2003.

FRANCO, Túlio Batista; MERHY, Emerson Elias. Programa de Saúde da Família (PSF): contradições de um programa destinado à mudança do modelo tecnoassistencial. In: MERHY, 183 Emerson Elias et al (org). O trabalho em saúde: olhando e experenciando o SUS no cotidiano. São Paulo: Ed. Hucitec, 2003. p.55-124.

MENDES, E. V. O Cuidado das Condições Crônicas na Atenção Primária à Saúde: O imperativo da Consolidação da Estratégia de Saúde da Família. Brasília: Organização PanAmericana da Saúde, 2012, 512 p.

MONNERAT, Giselle Lavinas; et al. Entre a formulação e a implementação: uma análise do Programa Saúde da Família. In: BRAVO, Maria Inês Souza, et al (Orgs). Saúde e Serviço Social. São Paulo: Cortez; Rio de Janeiro: UERJ, 2007. p. 97-116.

OSÓRIO L. C., Grupos-teorias e práticas- acessando a era da grupalidade- Porto Alegre: Artes Médicas, 2000.

PAIM, Jairnilson Silva. Modelos assistências: reformulando o pensamento e incorporando a proteção e a promoção da saúde. 2001.

ROSA, W. de A. G.; LABATE, R. C. Programa Saúde da família: A construção de um novo modelo de assistência. Revista Latino Americana de Enfermagem, Ribeirão Preto, v. 13, n.6, p. 01-10, nov./dez. 2005.

SCHRAIBER, L. B, MENDES, G. R. B. Necessidades de saúde e atenção primária. In: Schaiber LB, Nemes MIB, Mendes Gonçalves RB, editores. Saúde do adulto: programas e ações na unidade básica. (SP): Hucitec; 1998.

SILVA JUNIOR, Aluísio Gomes da; ALVES, Carla Almeida. Modelos Assistenciais em Saúde: desafios e perspectivas. In: MOROSINI, Marcia Valeria G. C; CORBO, Anamaria D'Andreia. (Orgs). Modelos de atenção e a saúde da família. Rio de Janeiro: EPSJV/FIOCRUZ, 2007. p.27- 42.

SILVA, Rafael Vieira Braga da; et al. Do Elo ao Laço: O agente comunitário de saúde na construção da integralidade em saúde. In: MATTOS, Ruben Araújo; PINHEIRO, Roseni (orgs). Cuidado as fronteiras da integralidade. Universidade Estadual do Rio de Janeiro. Instituto de Medicina Social, Rio de Janeiro, 2005. p. 75 - 90.

SISTEMA ÚNICO DE SAÚDE, 2008.

SOARES, Laura Tavares. Saúde para todos. São Paulo. 2005.

STARFIELD, B. Atenção primária: equilíbrio entre necessidades de saúde, serviços e Tecnologia. Brasilia: UNESCO/Ministério da Saúde, 2002. 726p. 
TEIXEIRA, Mary Jane Oliveira; NUNES, Sheila Torres. A interdisciplinaridade no programa saúde da família: uma utopia? In: BRAVO, Maria Inês Souza, et al (Orgs). Saúde e Serviço Social. São Paulo: Cortez; Rio de Janeiro: UERJ, 2007. p.117-134.

VIANA, A. L. D.; DAL POZ, M. R. A Reforma do sistema de saúde no Brasil e o Programa de Saúde da Família. Rev. Saúde coletiva, Rio de janeiro: Physis, 1998. v.8, n. 2, p. 11-48. 